\title{
Beneficial effect modification on survival outcome of sepsis between ART-123 and polymyxin B-immobilised haemoperfusion: a nationwide Japanese registry study
}

\author{
Katsunori Mochizuki* ${ }^{*}$, Kotaro Mori, Hiroshi Kamijo, Michitaro Ichikawa, Kenichi Nitta and Hiroshi Imamura
}

\begin{abstract}
Background: Although recently published randomised controlled trials did not confirm significant positive effect of ART-123 or polymyxin B-immobilised haemoperfusion (PMX-HP) on survival outcome, previous studies using a dataset of 3195 patients with sepsis registered at 42 intensive care units throughout Japan revealed significantly reduced mortality following these treatments. A study has suggested the efficacy of combination therapy with ART-123 and PMX-HP; however, it did not evaluate the effect modification between them. We hypothesised that coadministration of ART-123 and PMX-HP has a significant positive effect modification on survival outcome. The purpose of this study was to evaluate the effect modification between ART-123 and PMX-HP treatment on the survival outcome of sepsis using post hoc analysis of the dataset of the Japan Septic Disseminated Intravascular Coagulation registry.
\end{abstract}

Results: Of the 3195 patients recorded in the registry, 2350 were analysed. The product term between ART-123 and PMX-HP was analysed by the Cox regression model to evaluate significance. The primary outcome of this study was hospital mortality. Although the administration of ART-123 was independently positively associated with survival outcome (adjusted hazard ratio [HR]: 0.834, 95\% confidence interval [Cl] 0.695-0.999; $P=0.049$ ) in the model prior to the introduction of the product term, a significant effect modification on survival outcome was observed between the administration of ART-123 and PMX-HP treatment (adjusted HR: $0.667,95 \% \mathrm{Cl} 0.462-0.961 ; P=0.030$ ).

Conclusions: The main effect of the administration of ART-123 may be beneficial for survival outcome in patients with sepsis. In addition, a significant beneficial effect modification on survival outcome was observed between the administration of ART-123 and PMX-HP treatment.

Keywords: Sepsis, Thrombomodulin, ART-123, Polymyxin B-immobilised haemoperfusion, Effect modification

\section{Background}

The survival outcome of sepsis is improving with the adoption of standards for treatment, such as the Surviving Sepsis Campaign Guidelines [1-4]. However, mortality is still high, and sepsis, a complicated condition characterised by life-threatening organ dysfunction secondary to infections, remains an important worldwide

\footnotetext{
${ }^{*}$ Correspondence: kmochizuki@shinshu-u.ac.jp
}

Department of Emergency and Critical Care Medicine, Shinshu University School of Medicine, 3-1-1 Asahi, Matsumoto, Nagano 390-8621, Japan public health issue [5-7]. Along with the core treatment approaches for infections (antibiotic therapy and source control), various additional treatments to control pathophysiological pathways leading to organ dysfunction have been investigated, with the goal of reducing the morbidity and the mortality of sepsis $[6,8]$.

The coagulation pathway, which mediates coagulopathy and disseminated intravascular coagulation (DIC) in sepsis, has been one such research target. Coagulopathy is a complication of sepsis that causes organ dysfunction and leads to high mortality [9-13]. In addition, 
coagulopathy and sepsis adversely affect each other via crosstalk between coagulation and inflammation pathways [14]. Therefore, several anticoagulants expected to control coagulopathy and reduce the mortality of sepsis have been investigated [15-17]. ART-123 (recombinant human soluble thrombomodulin) is a novel anticoagulant that also has an anti-inflammatory effect [18]. Although the clinical efficacy of ART-123 in reducing the mortality of sepsis has been thoroughly investigated [19-24], its effects were not significant in a recently published phase 3 randomised controlled trial (RCT) known as the SCARLET trial [25].

Activation of the endotoxin pathway induces organ dysfunction and shock in patients with Gram-negative microorganism infections [26]. To control the systemic inflammatory response, endotoxin removal using polymyxin B-immobilised haemoperfusion (PMX-HP) has been attempted [27, 28]. PMX-HP therapy was expected not only to stabilise the shock response in the hyperinflammatory phase, but also to alleviate the subsequent immunosuppressive phase, known as immunoparalysis, which causes secondary infections and increased mortality $[29,30]$. However, although the clinical efficacy of the PMX-HP therapy in improving the survival outcome was initially expected [31], no significant mortality reduction was observed in larger, more recent RCTs [32, 33], as well as in the meta-analysis that included those RCTs [34].

Although recently published RCTs have not confirmed the significant positive effects of ART-123 and PMX-HP on sepsis survival outcomes, previous studies using a dataset of 3195 registered adult patients with sepsis revealed significant efficacy for these approaches in reducing hospital mortality $[35,36]$. It has been suggested that specific target populations may obtain survival benefits from these therapies [24, 37, 38]. Therefore, differences in the characteristics of the patients analysed in these studies compared to those enrolled in the RCTs may explain the conflicting results. However, because the retrospective design makes it difficult to completely eliminate factors that can affect outcomes, there might be other reasons for the discrepancies, such as effect modifications with other therapies. In a 22-patient, single-centre study in 2013, Yamato et al. [39] reported the efficacy of a combination therapy with ART-123 and PMX-HP for patients with septic shock accompanied by DIC. However, the study did not evaluate the effect modification between these therapies, because, generally, a large sample size is needed to reveal a significant effect modification. The above dataset, named the Japan Septic Disseminated Intravascular Coagulation (J-Septic DIC) registry, is a unique published dataset that includes many patients who received anticoagulant therapies for septic coagulopathy and/or blood purification for septic shock
[40]. The knowledge of effect modifications between these therapies, which cannot be assessed within a single RCT including only one of them, would be useful for further research and clinical decisions. Therefore, we hypothesised that there would be a significant effect modification between ART-123 and PMX-HP, which would affect survival outcomes in this dataset. In the present study, we evaluated the effect modification between the administration of ART-123 and treatment with PMXHP on survival outcome using the dataset of the J-Septic DIC registry.

\section{Methods}

\section{Study design, setting, and population}

This study was conducted as a post hoc analysis of a retrospective cohort dataset of consecutive adult patients who were admitted to 42 intensive care units (ICUs) in 40 institutions throughout Japan for treatment of sepsis between January 2011 and December 2013 (the J-Septic DIC registry) [40]. We evaluated the effect modification between the administration of ART-123 and treatment with PMX-HP on survival outcome in the nationwide registry. The primary outcome was hospital mortality at discharge.

Sepsis manifestations in the registry were defined as "severe sepsis" and "septic shock" based on the conventional criteria proposed by the American College of Chest Physicians/Society of Critical Care Medicine consensus conference in 1991 [41]. Patients who were 18 years of age or older and had severe sepsis or septic shock at ICU admission were enrolled in the registry. In the present study, we excluded patients who had missing data in analysed variables, such as body weight, severity scores at ICU admission, blood lactate level on day 1, and data related to treatment (Fig. 1). Acute Physiology and Chronic Health Evaluation (APACHE) II, Sequential Organ Failure Assessment (SOFA), systemic inflammatory response syndrome (SIRS), and Japanese Association for Acute Medicine (JAAM)-DIC scores [42, 43] were used to measure severity. The JAAM-DIC score was calculated from the SIRS score, platelet count, prothrombin time-international normalised ratio, and level of fibrin/fibrinogen degradation product or D-dimer on day 1 . The JAAM-DIC score was considered missing if the patient had no data for any variables used in the score calculation.

\section{Analysed data}

We analysed the following variables collected in the J-Septic DIC registry as indicated in Table 1: patient characteristics, including ICU characteristics, severity score on day 1 , blood lactate level on day 1 , blood culture results, and primary infection site; therapeutic variables, 


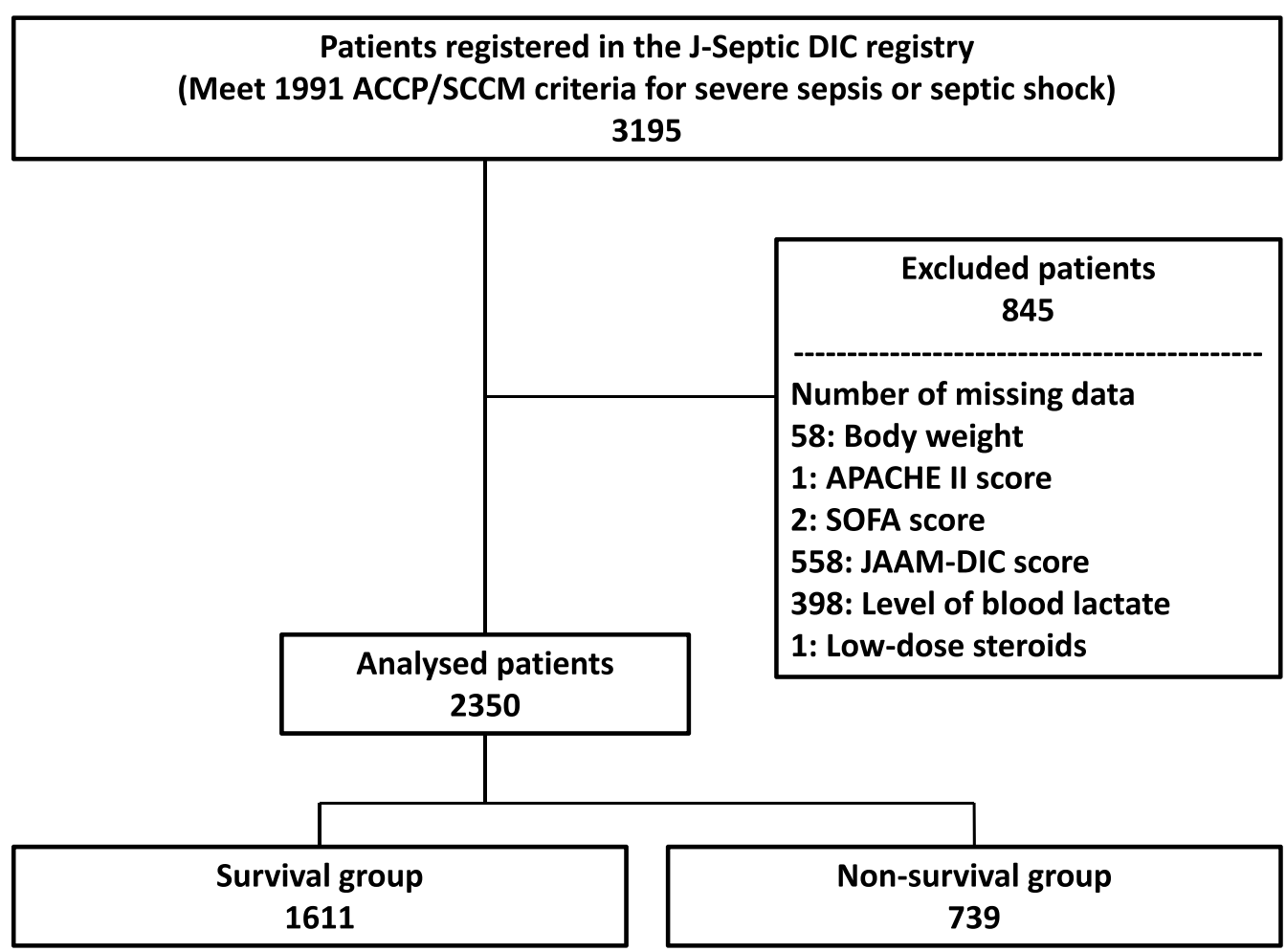

Fig. 1 Study flowchart. The numbers of patients are indicated in each box. ACCP American College of Chest Physicians, APACHE Acute Physiology and Chronic Health Evaluation, DIC disseminated intravascular coagulation, JAAM Japanese Association for Acute Medicine, J-Septic DIC Japan Septic Disseminated Intravascular Coagulation, SCCM Society of Critical Care Medicine, SOFA Sequential Organ Failure Assessment

including specific treatments, administration of anticoagulant for DIC treatment and anti-thrombotic drugs to treat conditions other than DIC during the first 7 days after ICU admission, and blood purifications during the first 7 days after ICU admission. Analysed outcome variables included bleeding complications (bleeding requiring transfusion, intracranial haemorrhage, bleeding requiring therapeutic intervention, and bleeding to death), days from ICU admission to hospital discharge, and hospital mortality at discharge. Age, body weight, severity scores, blood lactate levels, ventilator days, and days from ICU admission to hospital discharge were analysed as numerical variables, whereas other parameters were analysed as categorical variables.

\section{Statistical analysis}

The survival and nonsurvival groups were compared in terms of their patient characteristics, therapeutic variables, and outcome variables. Categorical variables were compared using the Chi squared and Fisher's exact tests, whereas numerical variables were compared using the Mann-Whitney U test. Categorical variables were presented as numbers and percentages, whereas numerical variables were summarised using the median and interquartile range (IQR).

The significance of effect modification between the administration of ART-123 and treatment with PMXHP was evaluated using the multivariate Cox regression model until day 90 . The product term was inputted into the Cox regression model for hospital mortality adjustment with most analysed patient characteristics and therapeutic variables as covariates. The presence of haemostatic disorders caused by liver cirrhosis was excluded as a variable because of the concerns about collinearity with the presence of chronic liver failure. In addition, the therapeutic variables veno-arterial and veno-venous extracorporeal membrane oxygenation, intra-aortic balloon pumping, as well as warfarin and other drug use for conditions other than DIC were excluded from the Cox regression model, because log-log plots of these variables revealed unsatisfied proportional hazard assumption of these variables. Furthermore, a subgroup analysis of patients who required vasopressors was performed using a similar Cox regression model to evaluate the significance of the effect modification between the administration of ART-123 and treatment with PMX-HP in shock-suspected patients. 
Table 1 Patient characteristics, therapies, and outcomes in the survival and nonsurvival groups

\begin{tabular}{|c|c|c|c|}
\hline & $\begin{array}{l}\text { Survival } \\
(n=1611)\end{array}$ & $\begin{array}{l}\text { Nonsurvival } \\
(n=739)\end{array}$ & $P$ value \\
\hline ICU management policy & & & 0.034 \\
\hline Closed, $n(\%)$ & $939(58.3)$ & $459(62.1)$ & \\
\hline Open, $n(\%)$ & $386(24.0)$ & $180(24.4)$ & \\
\hline Other, $n(\%)$ & $286(17.8)$ & $100(13.5)$ & \\
\hline Admission route to the ICU & & & $<0.001$ \\
\hline Emergency department, $n$ (\%) & $700(43.5)$ & $299(40.5)$ & \\
\hline Other hospital, $n$ (\%) & $517(32.1)$ & $167(22.6)$ & \\
\hline Ward, $n(\%)$ & $394(24.5)$ & $273(36.9)$ & \\
\hline Age (years) & $71(60,79)$ & $73(64,80)$ & $<0.001$ \\
\hline Male sex, $n(\%)$ & $947(58.8)$ & $469(63.5)$ & 0.031 \\
\hline Body weight (kg) & $55.7(47.8,65.0)$ & $54.2(47.0,63.0)$ & 0.008 \\
\hline \multicolumn{4}{|c|}{ Pre-existing organ insufficiency or immunosuppression based on APACHE II score } \\
\hline Liver, $n(\%)$ & $48(3.0)$ & $61(8.3)$ & $<0.001$ \\
\hline Respiratory, $n(\%)$ & $54(3.4)$ & $40(5.4)$ & 0.018 \\
\hline Cardiovascular, $n(\%)$ & $78(4.8)$ & $67(9.1)$ & $<0.001$ \\
\hline Renal, $n(\%)$ & $95(5.9)$ & $86(11.6)$ & $<0.001$ \\
\hline Immunocompromised, $n$ (\%) & $202(12.5)$ & $170(23.0)$ & $<0.001$ \\
\hline \multicolumn{4}{|l|}{ Pre-existing haemostatic disorders } \\
\hline Cirrhosis, $n(\%)$ & $48(3.0)$ & $55(7.4)$ & $<0.001$ \\
\hline Haematological malignancy, $n(\%)$ & $31(1.9)$ & $48(6.5)$ & $<0.001$ \\
\hline Chemotherapy, n (\%) & $48(3.0)$ & $61(8.3)$ & $<0.001$ \\
\hline Warfarin intake, $n(\%)$ & $71(4.4)$ & $30(4.1)$ & 0.700 \\
\hline Other, $n(\%)$ & $23(1.4)$ & $26(3.5)$ & 0.001 \\
\hline APACHE II score & $21(16,26)$ & $28(21,35)$ & $<0.001$ \\
\hline SOFA score & $9(6,11)$ & $12(9,15)$ & $<0.001$ \\
\hline SIRS score & $3(2,4)$ & $3(2,4)$ & 0.031 \\
\hline JAAM-DIC score & $3(2,5)$ & $5(3,6)$ & $<0.001$ \\
\hline Blood lactate $(\mathrm{mmol} / \mathrm{L})$ & $2.6(1.6,4.6)$ & $4.5(2.1,8.9)$ & $<0.001$ \\
\hline Blood culture & & & $<0.001$ \\
\hline Not taken, $n(\%)$ & $87(5.4)$ & $23(3.1)$ & \\
\hline Positive, $n(\%)$ & $659(40.9)$ & $364(49.3)$ & \\
\hline Negative, $n(\%)$ & $865(53.7)$ & $352(47.6)$ & \\
\hline Microorganisms & & & 0.033 \\
\hline Unknown, $n(\%)$ & $352(21.8)$ & $150(20.3)$ & \\
\hline Virus, $n(\%)$ & $14(0.9)$ & $7(0.9)$ & \\
\hline Gram-negative rod, $n$ (\%) & $606(37.6)$ & $239(32.3)$ & \\
\hline Gram-positive coccus, n (\%) & 381 (23.6) & $185(25.0)$ & \\
\hline Fungus, $n(\%)$ & $25(1.6)$ & $16(2.2)$ & \\
\hline Mixed infection, $n(\%)$ & $203(12.6)$ & $127(17.2)$ & \\
\hline Others, $n(\%)$ & $30(1.9)$ & $15(2.0)$ & \\
\hline Primary source of infection & & & $<0.001$ \\
\hline Unknown, $n(\%)$ & $75(4.7)$ & $69(9.3)$ & \\
\hline Catheter-related bloodstream infection, $n$ (\%) & $17(1.1)$ & $12(1.6)$ & \\
\hline Bone or soft tissue, $n(\%)$ & $220(13.7)$ & $80(10.8)$ & \\
\hline Cardiovascular system, n (\%) & $33(2.0)$ & $12(1.6)$ & \\
\hline Central nervous system, $n$ (\%) & $34(2.1)$ & $18(2.4)$ & \\
\hline Urinary tract, $n(\%)$ & $295(18.3)$ & $63(8.5)$ & \\
\hline Lung or thoracic cavity, n (\%) & $366(22.7)$ & $249(33.7)$ & \\
\hline Abdomen, $n(\%)$ & $541(33.6)$ & $228(30.9)$ & \\
\hline
\end{tabular}


Table 1 (continued)

\begin{tabular}{|c|c|c|c|}
\hline & $\begin{array}{l}\text { Survival } \\
(n=1611)\end{array}$ & $\begin{array}{l}\text { Nonsurvival } \\
(n=739)\end{array}$ & $P$ value \\
\hline Other, $n(\%)$ & $30(1.9)$ & $8(1.1)$ & \\
\hline \multicolumn{4}{|l|}{ Specific treatments } \\
\hline Surgical intervention, $n(\%)$ & $740(45.9)$ & $250(33.8)$ & $<0.001$ \\
\hline Mechanical ventilator, (days) & $4(0,9)$ & $5(2,16)$ & $<0.001$ \\
\hline Vasopressor, $n(\%)$ & $1166(72.4)$ & $663(89.7)$ & $<0.001$ \\
\hline Immunoglobulins, $n(\%)$ & $520(32.3)$ & $271(36.7)$ & 0.036 \\
\hline Low-dose steroids, $n$ (\%) & $330(20.5)$ & $286(38.7)$ & $<0.001$ \\
\hline Veno-arterial ECMO, n (\%) & $5(0.3)$ & $18(2.4)$ & $<0.001$ \\
\hline Veno-venous ECMO, $n$ (\%) & $15(0.9)$ & $19(2.6)$ & 0.002 \\
\hline Intra-aortic balloon pumping, $n(\%)$ & $4(0.2)$ & $6(0.8)$ & 0.081 \\
\hline \multicolumn{4}{|l|}{ Therapeutic interventions for DIC } \\
\hline ART-123, n (\%) & $489(30.4)$ & $231(31.3)$ & 0.659 \\
\hline Antithrombin, $n(\%)$ & $541(33.6)$ & $279(37.8)$ & 0.049 \\
\hline Protease inhibitors, $n$ (\%) & $185(11.5)$ & $120(16.2)$ & 0.001 \\
\hline Heparinoids, $n$ (\%) & $85(5.3)$ & $36(4.9)$ & 0.680 \\
\hline \multicolumn{4}{|l|}{ Antithrombotic drugs for conditions other than DIC } \\
\hline Heparin, $n(\%)$ & $210(13.0)$ & $87(11.8)$ & 0.392 \\
\hline Warfarin, $n(\%)$ & $23(1.4)$ & $4(0.5)$ & 0.061 \\
\hline Anti-platelet drugs, $n(\%)$ & $35(2.2)$ & $13(1.8)$ & 0.511 \\
\hline Other, $n(\%)$ & $12(0.7)$ & $3(0.4)$ & 0.415 \\
\hline Nafamostat mesylate for blood purifications, $n(\%)$ & $398(24.7)$ & $298(40.3)$ & $<0.001$ \\
\hline \multicolumn{4}{|l|}{ Blood purifications } \\
\hline $\mathrm{PMX}-\mathrm{HP}, n(\%)$ & $332(20.6)$ & $189(25.6)$ & 0.007 \\
\hline RRT, $n(\%)$ & $369(22.9)$ & $327(44.2)$ & $<0.001$ \\
\hline RRT for non-renal indications, $n(\%)$ & $115(7.1)$ & $80(10.8)$ & 0.003 \\
\hline Plasma exchange, $n(\%)$ & $8(0.5)$ & $15(2.0)$ & $<0.001$ \\
\hline Concomitant treatment with ART-123 and PMX-HP, n (\%) & $164(10.2)$ & $83(11.2)$ & 0.440 \\
\hline Bleeding complications, $n$ (\%) & $155(9.6)$ & $129(17.5)$ & $<0.001$ \\
\hline Time from ICU admission to hospital discharge (days) & $33(18,61)$ & $14(3,30.5)$ & $<0.001$ \\
\hline
\end{tabular}

Data are presented as $n$ (\%) or median (interquartile range)

$A P A C H E$ acute physiology and chronic health evaluation, DIC disseminated intravascular coagulation, ECMO extracorporeal membrane oxygenation, ICU intensive care unit, JAAM Japanese Association for Acute Medicine, PMX-HP polymyxin B-immobilised haemoperfusion, RRT renal replacement therapy, SIRS systemic inflammatory response syndrome, SOFA sequential organ failure assessment

We did not impute any missing data and performed a complete case analysis for all analyses. All statistical analyses were performed using IBM SPSS Statistics version 26 (IBM Co., Armonk, New York, USA) and differences were considered statistically significant if $P<0.05$.

\section{Results}

\section{Patient characteristics in the survival and nonsurvival groups}

Of the 3195 patients in the J-Septic DIC registry, 2350 patients were included in the final analysis after the exclusion of 845 patients that missed data for any of the analysed variables (Fig. 1). The median patient age was 71 years (IQR: 62, 80 years) and 60.3\% (1416/2350) of the patients were male. On ICU admission day, the median APACHE II, SOFA, SIRS, and JAAM-DIC scores were 23 (IQR: 17, 29), 10 (IQR: 7, 13), 3 (IQR: 2, 4), and 4 (IQR: 2, $6)$, respectively. The rate of hospital mortality was $31.4 \%$ (739/2350).

Table 1 lists patient characteristics, therapeutic variables, and outcome variables of the survival and nonsurvival groups. Patient age and severity scores were significantly higher in the nonsurvival group, and bleeding complications were more frequently observed $(17.5 \%$ vs. $9.6 \% ; P<0.001)$. Among the variables evaluated for effect modification, PMX-HP treatment was more frequent in the nonsurvival group $(25.6 \%$ vs. $20.6 \% ; P=0.007$ ), whereas the proportions of patients 
that received ART-123 were not significantly different between the two groups $(31.3 \%$ vs. $30.4 \%$; $P=0.659)$.

\section{Effect modification of combined ART-123/PMX-HP treatment on survival outcome}

Table 2 shows the covariate-adjusted Cox regression model. Prior to the introduction of the product term, the administration of ART-123 was independently associated with the survival outcome (adjusted hazard ratio [HR]: 0.834, 95\% confidence interval [CI] 0.695-0.999; $P=0.049$ ). Table 3 shows the adjusted $\mathrm{HR}, 95 \% \mathrm{CI}$, and $P$ values of the product term between ART-123 and PMX$\mathrm{HP}$, and related therapeutic variables after the product term was introduced into the Cox regression model. The effect modification between the administration of ART123 and PMX-HP treatment significantly affected the survival outcome (adjusted HR: 0.667, 95\% CI 0.462$0.961 ; P=0.030$ ) (Table 3a).

Table 4 shows the covariate-adjusted Cox regression model for the subgroup of 1829 patients who required vasopressors. The effect modification between ART-123 administration and PMX-HP treatment significantly affected the survival outcome in the subgroup (adjusted HR: $0.637,95 \%$ CI $0.439-0.925 ; P=0.018$ ) (Table 3b).

\section{Discussion}

Our results demonstrate that the main effect of the administration of ART-123 may be beneficial for survival outcome, and its effects were augmented by a significant effect modification upon co-treatment with PMX-HP. This study is the first to demonstrate a significant positive effect modification between the administration of ART-123 and treatment with PMX-HP on the survival outcome of patients with sepsis.

In 2016, Hayakawa et al. [35] used propensity score analysis to analyse J-Septic DIC registry data, and reported a significantly improved survival outcome following ART-123 treatment in patients with sepsisinduced DIC. In that report, survival times between propensity score-matched ART-123 and control groups were significantly different (HR: $0.781,95 \%$ CI 0.624-0.977; $P=0.030$ ). In this study, although the inclusion criteria and statistical model used were different, the main effect of ART-123 administration, before adjusting for product terms, was also significantly beneficial to survival (adjusted HR: 0.834, 95\% CI 0.695-0.999; $P=0.049$ ). However, we also observed a significant effect modification between treatments with ART-123 and PMX-HP. Thus, the effect of ART-123 observed in the study by Hayakawa et al. may also have been influenced by that effect modification. In that study, $31.6 \%$ of patients in the ART123 group after propensity score matching also received PMX-HP therapy. In addition, in 2017, Nakamura et al.
[36] reported a significant positive effect of the PMXHP therapy on survival outcome using the same dataset and propensity score matching. In their study, they used a different indicator for survival outcome (the odds ratio for hospital mortality) in the population different from that in the present study, making the comparison of the results of these two studies complicated. However, 38.9\% of patients in the PMX-HP group after propensity score matching received ART-123, thus the effect of PMX-HP observed in the study by Nakamura et al. [36] might also have been influenced by this effect modification. Propensity score matching and other propensity score analyses can be useful to control biases in observational studies [44]; however, the bias reducing capabilities of propensity scores may decrease when the propensity scores are estimated without considering interactions [45].

The mechanism of the effect modification between ART-123 and PMX-HP treatments remains unclear. Although the predominant effect of PMX-HP is thought to be endotoxin removal, it has also been reported that PMX-HP traps activated leukocytes and platelets [28]. Activated blood cells are known to mediate the development of coagulopathy, which is followed by organ dysfunction and shock during sepsis. Iba et al. [46] suggested that the adsorption of such activated blood cells might be a therapeutic strategy against the complex mechanism of shock development during sepsis, in which the coagulation pathway plays an important role. Yamato et al. [39] reported efficacy for ART-123/PMX-HP combination therapy in patients with septic shock accompanied by DIC, suggesting that simultaneous control of high-mobility group box-1 protein, a late mediator of sepsis, through ART-123 and PMX-HP therapy might be a putative mechanism underpinning the beneficial effect. Although the present study could not reveal the detailed mechanism of the effect modification between ART-123 and PMX-HP treatments, our analysis of a large multicentre sample of 2350 patients supports the possibility of clinical efficacy of the combination reported by Yamato et al. We believe that in addition to the independent primary mechanisms of each therapy (anticoagulation and endotoxin removal), the simultaneous targeting of multiple mediators related to the development of organ dysfunction and shock in sepsis likely explains the effect modification between these therapies.

The use of a Japanese nationwide dataset, which included patients that received several novel interventions for sepsis, was a particular strength of the present study. Analysis of effect modifications is difficult to perform with a small sample size dataset, because the sample size of each variable evaluated for the effect modifications is smaller than overall sample size. In this study, ART-123 was administered to 720 patients 
Table 2 Cox regression model adjusted for patient characteristics and therapeutic variables for hospital mortality

\begin{tabular}{|c|c|c|c|}
\hline & Adjusted hazard ratio & $95 \%$ confidence interval & $P$ value \\
\hline \multicolumn{4}{|l|}{ ICU management policy } \\
\hline Closed & Reference & & \\
\hline Open & 1.113 & $0.919-1.348$ & 0.275 \\
\hline Other & 0.744 & $0.584-0.947$ & 0.017 \\
\hline \multicolumn{4}{|l|}{ Admission route to the ICU } \\
\hline Emergency department & Reference & & \\
\hline Another hospital & 0.877 & $0.713-1.078$ & 0.211 \\
\hline Ward & 0.981 & $0.812-1.187$ & 0.847 \\
\hline Age (years) & 1.013 & $1.006-1.020$ & $<0.001$ \\
\hline Male sex & 1.125 & $0.947-1.337$ & 0.179 \\
\hline Body weight (kg) & 0.991 & $0.984-0.997$ & 0.004 \\
\hline \multicolumn{4}{|c|}{ Pre-existing organ insufficiency or immunosuppression based on APACHE II score } \\
\hline Liver & 1.278 & $0.952-1.716$ & 0.102 \\
\hline Respiratory & 1.422 & $1.020-1.983$ & 0.038 \\
\hline Cardiovascular & 1.354 & $1.024-1.790$ & 0.034 \\
\hline Renal & 1.460 & $1.132-1.885$ & 0.004 \\
\hline Immunocompromised & 1.081 & $0.864-1.352$ & 0.497 \\
\hline \multicolumn{4}{|l|}{ Pre-existing haemostatic disorders } \\
\hline Haematological malignancy & 1.118 & $0.771-1.619$ & 0.556 \\
\hline Chemotherapy & 0.982 & $0.710-1.360$ & 0.915 \\
\hline Warfarin intake & 0.776 & $0.524-1.150$ & 0.206 \\
\hline Other & 1.464 & $0.939-2.283$ & 0.093 \\
\hline APACHE II score & 1.035 & $1.023-1.047$ & $<0.001$ \\
\hline SOFA score & 1.088 & $1.054-1.123$ & $<0.001$ \\
\hline SIRS score & 0.953 & $0.869-1.047$ & 0.316 \\
\hline JAAM-DIC score & 1.032 & $0.987-1.079$ & 0.161 \\
\hline Blood lactate (mmol/L) & 1.083 & $1.066-1.100$ & $<0.001$ \\
\hline \multicolumn{4}{|l|}{ Blood culture } \\
\hline Not taken & Reference & & \\
\hline Positive & 1.083 & $0.690-1.700$ & 0.728 \\
\hline Negative & 0.885 & $0.573-1.365$ & 0.581 \\
\hline \multicolumn{4}{|l|}{ Microorganisms } \\
\hline Unknown & Reference & & \\
\hline Virus & 0.945 & $0.400-2.231$ & 0.898 \\
\hline Gram-negative rod & 0.791 & $0.612-1.023$ & 0.074 \\
\hline Gram-positive coccus & 0.902 & $0.685-1.188$ & 0.463 \\
\hline Fungus & 1.158 & $0.644-2.083$ & 0.625 \\
\hline Mixed infection & 1.034 & $0.784-1.364$ & 0.810 \\
\hline Others & 1.126 & $0.642-1.975$ & 0.678 \\
\hline \multicolumn{4}{|l|}{ Primary source of infection } \\
\hline Unknown & Reference & & \\
\hline Catheter-related bloodstream infection & 0.631 & $0.317-1.254$ & 0.189 \\
\hline Bone or soft tissue & 0.765 & $0.525-1.116$ & 0.165 \\
\hline Cardiovascular system & 0.566 & $0.289-1.109$ & 0.097 \\
\hline Central nervous system & 0.579 & $0.328-1.023$ & 0.060 \\
\hline Urinary tract & 0.538 & $0.364-0.794$ & 0.002 \\
\hline Lung or thoracic cavity & 1.084 & $0.803-1.464$ & 0.598 \\
\hline Abdomen & 0.774 & $0.555-1.079$ & 0.131 \\
\hline Other & 0.625 & $0.290-1.348$ & 0.231 \\
\hline
\end{tabular}


Table 2 (continued)

\begin{tabular}{|c|c|c|c|}
\hline & Adjusted hazard ratio & 95\% confidence interval & $P$ value \\
\hline \multicolumn{4}{|l|}{ Specific treatments } \\
\hline Surgical intervention & 0.766 & $0.619-0.948$ & 0.014 \\
\hline Mechanical ventilator (days) & 0.977 & $0.967-0.987$ & $<0.001$ \\
\hline Vasopressor & 1.290 & $0.979-1.701$ & 0.070 \\
\hline Immunoglobulins & 0.859 & $0.719-1.027$ & 0.095 \\
\hline Low-dose steroids & 1.420 & $1.190-1.695$ & $<0.001$ \\
\hline \multicolumn{4}{|l|}{ Therapeutic interventions for DIC } \\
\hline ART-123 & 0.834 & $0.695-0.999$ & 0.049 \\
\hline Antithrombin & 0.875 & $0.728-1.053$ & 0.158 \\
\hline Protease inhibitors & 0.916 & $0.727-1.152$ & 0.452 \\
\hline Heparinoids & 1.014 & $0.706-1.457$ & 0.940 \\
\hline \multicolumn{4}{|c|}{ Anti-thrombotic drugs for conditions other than DIC } \\
\hline Heparin & 0.690 & $0.537-0.886$ & 0.004 \\
\hline Anti-platelet drugs & 0.646 & $0.342-1.221$ & 0.179 \\
\hline Nafamostat mesylate for blood purifications & 0.702 & $0.553-0.889$ & 0.003 \\
\hline \multicolumn{4}{|l|}{ Blood purifications } \\
\hline PMX-HP & 0.897 & $0.720-1.118$ & 0.333 \\
\hline RRT & 1.383 & $1.090-1.756$ & 0.008 \\
\hline RRT for non-renal indications & 1.300 & $0.992-1.704$ & 0.057 \\
\hline Plasma exchange & 1.498 & $0.838-2.677$ & 0.172 \\
\hline
\end{tabular}

APACHE acute physiology and chronic health evaluation, DIC disseminated intravascular coagulation, ECMO extracorporeal membrane oxygenation, ICU intensive care unit, JAAM Japanese Association for Acute Medicine, PMX-HP polymyxin B-immobilised haemoperfusion, RRT renal replacement therapy, SIRS systemic inflammatory response syndrome, SOFA sequential organ failure assessment

Table 3 Adjusted hazard ratios of product terms between ART-123 and PMX-HP and related therapeutic variables

\begin{tabular}{|c|c|c|c|}
\hline & $\begin{array}{l}\text { Adjusted } \\
\text { hazard ratio }\end{array}$ & $\begin{array}{l}95 \% \text { confidence } \\
\text { interval }\end{array}$ & $P$ value \\
\hline \multicolumn{4}{|l|}{ (a) Overall } \\
\hline ART-123 $\times$ PMX-HP & 0.667 & $0.462-0.961$ & 0.030 \\
\hline ART-123 & 0.774 & $0.639-0.937$ & 0.009 \\
\hline PMX-HP & 0.872 & $0.699-1.086$ & 0.222 \\
\hline \multicolumn{4}{|c|}{ (b) Patients who required vasopressors } \\
\hline ART-123 × PMX-HP & 0.637 & $0.439-0.925$ & 0.018 \\
\hline ART-123 & 0.790 & $0.649-0.960$ & 0.018 \\
\hline PMX-HP & 0.889 & $0.710-1.112$ & 0.301 \\
\hline
\end{tabular}

PMX-HP polymyxin B-immobilised haemoperfusion

The adjusted hazard ratio, 95\% confidence interval, and $P$ values indicate those calculated after the product term was introduced into the Cox regression model shown in Table 2 or 4

(30.6\% of the total cohort), PMX-HP was performed in 521 patients (22.2\% of the total cohort), and concomitant therapy was administered to 247 patients $(10.5 \%$ of the total cohort, $34.3 \%$ of patients who received ART-123, and $47.4 \%$ of the patients who received PMX-HP). In Japan, ART-123 was approved for the indication of DIC by the Ministry of Health, Labour and Welfare in 2008
[47], whereas PMX-HP treatment was approved for the indication of severe Gram-negative bacterial infection in 1994 [27]. To the best of our knowledge, it is only in Japan that both these therapies can be used in general clinical setting. Thus, the nationwide dataset compiled in Japan, which comprised hundreds of patients who received unique treatments for sepsis, was useful for the evaluation of effect modification between these relatively novel approaches. It should be noted that numerous RCTs designed to evaluate the effects of each individual therapy in comparison to the standard of care [20-22, 25, 31-33] could not examine effect modifications between the novel treatments.

This study has several limitations. First, its retrospective observational design is associated with a risk of unmeasured or unknown biases. Second, approximately a quarter of eligible patients were excluded because of missing data for some of the analysed variables. This selection process might also have introduced the risk of bias. However, there were several analysed variables that were not reported in over $10 \%$ of patients; therefore, we did not use the multiple imputation method. Third, the J-Septic DIC dataset is relatively old, and the definitions of severe sepsis and septic shock used in the dataset were proposed in 1991 [41], whereas the current definitions of sepsis and septic shock were published in 2016 
Table 4 Cox regression model adjusted for patient characteristics and therapeutic variables to assess hospital mortality in patients who received vasopressors

ICU management policy

Closed
Open
Other

Admission route to the $\mathrm{ICU}$

Emergency department

Another hospital

Ward

Age (years)

Male sex

Body weight $(\mathrm{kg})$

Pre-existing organ insufficiency or immunosuppression based on APACHE 1.210
Liver

Respiratory

Cardiovascular

Renal

Immunocompromised

Pre-existing haemostatic disorders

Haematological malignancy

Chemotherapy

Warfarin intake

Blood lactate $(\mathrm{mmol} / \mathrm{L})$

Blood culture

Not taken

Positive

Negative

Microorganisms

Unknown

Virus

Gram-negative rod

Gram-positive coccus

Fungus

Mixed infection

Others

Primary source of infection

Unknown

Catheter-related bloodstream infection

Bone or soft tissue

Cardiovascular system

Central nervous system

Urinary tract

Lung or thoracic cavity

Abdomen

Other
Adjusted hazard ratio

Reference

1.076

0.746

Reference

0.858

0.965

1.014

1.219

0.992

ACHE II score

1.412

1.413

1.467

1.088

1.070

0.981

0.807

1.202

1.037

1.078

0.958

1.029

1.083

$95 \%$ confidence interval

$P$ value

$0.876-1.320$

0.486

$0.579-0.962$

0.024

$0.689-1.069$

0.172

$0.791-1.178$

0.727

1.007-1.022

$<0.001$

$1.015-1.464$

0.034

0.986-0.999

0.027

0.888-1.649

0.228

$0.997-1.998$

0.052

$1.061-1.883$

0.018

0.005

0.479

0.720-1.591

0.737

0.699-1.377

0.912

0.296

0.486

$<0.001$

$<0.001$

0.400

0.226

$<0.001$

Reference

1.022

$0.623-1.678$

0.930

0.813

$0.504-1.313$

0.398

Reference

0.692

0.800

0.939

1.245

1.031

1.061

$0.245-1.956$

0.488

0.607-1.052

0.111

0.699-1.262

0.678

$0.683-2.269$

0.474

$0.764-1.390$

0.843

0.566-1.990

0.853

Reference

0.653

0.816

0.577

0.708

0.544

1.114

0.784

0.632

$\begin{array}{ll}0.325-1.312 & 0.232 \\ 0.547-1.218 & 0.320 \\ 0.284-1.171 & 0.128 \\ 0.379-1.323 & 0.279 \\ 0.357-0.830 & 0.005 \\ 0.807-1.537 & 0.512 \\ 0.551-1.116 & 0.176 \\ 0.277-1.439 & 0.274\end{array}$


Table 4 (continued)

\begin{tabular}{|c|c|c|c|}
\hline & Adjusted hazard ratio & $95 \%$ confidence interval & $P$ value \\
\hline \multicolumn{4}{|l|}{ Specific treatments } \\
\hline Surgical intervention & 0.767 & $0.614-0.958$ & 0.019 \\
\hline Mechanical ventilator (days) & 0.974 & $0.964-0.985$ & $<0.001$ \\
\hline Immunoglobulins & 0.885 & $0.735-1.066$ & 0.198 \\
\hline Low-dose steroids & 1.387 & $1.154-1.666$ & $<0.001$ \\
\hline \multicolumn{4}{|l|}{ Therapeutic interventions for DIC } \\
\hline ART-123 & 0.849 & $0.704-1.025$ & 0.089 \\
\hline Antithrombin & 0.885 & $0.730-1.072$ & 0.210 \\
\hline Protease inhibitors & 0.893 & $0.702-1.137$ & 0.358 \\
\hline Heparinoids & 1.045 & $0.709-1.542$ & 0.823 \\
\hline \multicolumn{4}{|l|}{ Anti-thrombotic drugs for conditions other than DIC } \\
\hline Heparin & 0.696 & $0.536-0.904$ & 0.007 \\
\hline Anti-platelet drugs & 0.706 & $0.361-1.382$ & 0.310 \\
\hline Nafamostat mesylate for blood purifications & 0.699 & $0.547-0.893$ & 0.004 \\
\hline \multicolumn{4}{|l|}{ Blood purifications } \\
\hline PMX-HP & 0.915 & $0.731-1.146$ & 0.440 \\
\hline RRT & 1.398 & $1.092-1.789$ & 0.008 \\
\hline RRT for non-renal indications & 1.323 & $1.005-1.740$ & 0.046 \\
\hline Plasma exchange & 1.632 & $0.864-3.083$ & 0.131 \\
\hline
\end{tabular}

APACHE acute physiology and chronic health evaluation, DIC disseminated intravascular coagulation, ECMO extracorporeal membrane oxygenation, ICU intensive care unit, JAAM Japanese Association for Acute Medicine, PMX-HP polymyxin B-immobilised haemoperfusion, RRT renal replacement therapy, SIRS systemic inflammatory response syndrome, SOFA sequential organ failure assessment

[5], after the J-Septic DIC registry was compiled. Fourth, we used the JAAM-DIC score, which is predominantly used in Japan, and our conclusions might not extend to hospitals that use the International Society of Thrombosis and Haemostasis (ISTH) criteria [48]. However, we found that the JAAM-DIC score diagnosed most of the overt DIC cases, as do the ISTH criteria [42, 43, 49, 50]. In addition, 911 patients (28.5\%) had missing data in variables necessary for the calculation of the ISTH criteria. Therefore, we reasoned that it would be difficult to use the ISTH score in the present study, as it would require adjustments to many factors in the Cox regression model. Fifth, in Japan, continuous intracircuit infusion of nafamostat mesylate (NM) may be used as an anticoagulant treatment during PMX-HP. Because the main effect of $\mathrm{NM}$ was significant in the Cox regression model used in the present study, there is a possibility that the results were affected by NM infusion during PMX-HP. However, no significant effect modification between ART-123 and NM treatments was observed (data not shown). Sixth, we did not evaluate simple main effects of ART-123 and PMX-HP, because the subgroups did not have adequate sample size to be evaluated by the Cox regression model used in the present study. Further studies will be needed to validate our findings; however, the results of the present study might help designing optimal RCTs to evaluate the effects of ART-123 and/or PMX-HP and impact clinical decision-making.

\section{Conclusion}

A significant beneficial effect modification on survival outcome between the administration of ART-123 and PMX-HP treatment was observed in patients with sepsis. Further study is needed to evaluate the effects of combination therapy with ART-123 and PMX-HP on survival outcomes.

\section{Abbreviations \\ APACHE: Acute Physiology and Chronic Health Evaluation; Cl: Confidence interval; DIC: Disseminated intravascular coagulation; HR: Hazard ratio; ICU: Intensive care unit; IQR: Interquartile range; JAAM: Japanese Association for Acute Medicine; J-Septic DIC: Japan Septic Disseminated Intravascular Coagu- lation; PMX-HP: Polymyxin B-immobilised haemoperfusion; RCT: Randomised controlled trial; RRT: Renal replacement therapy; SIRS: Systemic inflammatory response syndrome; SOFA: Sequential Organ Failure Assessment.}

\section{Acknowledgements}

We would like to thank Editage (http://www.editage.jp) for English language editing. We greatly appreciate the J-Septic DIC study investigators (Mineji Hayakawa, Kazuma Yamakawa, Shinjiro Saito, Shigehiko Uchino, Daisuke Kudo, Yusuke lizuka, Masamitsu Sanui, Kohei Takimoto, Toshihiko Mayumi, Takeo Azuhata, Fumihito Ito, Shodai Yoshihiro, Hayakawa Katsura, Tsuyoshi Nakashima, Takayuki Ogura, Eiichiro Noda, Yoshihiko Nakamura, Ryosuke Sekine, Yoshiaki Yoshikawa, Motohiro Sekino, Keiko Ueno, Yuko Okuda, Masayuki Watanabe, Akihito Tampo, Nobuyuki Saito, Yuya Kitai, Hiroki Takahashi, Iwao Kobayashi, Yutaka Kondo, Wataru Matsunaga, Sho Nachi, Toru Miike, Hiroshi Takahashi, 
Shuhei Takauji, Kensuke Umakoshi, Takafumi Todaka, Hiroshi Kodaira, Kohkichi Andoh, Takehiko Kasai, Yoshiaki Iwashita, Hideaki Arai, Masato Murata, Masahiro Yamane, Kazuhiro Shiga, and Naoto Hori) for publishing the dataset.

\section{Authors' contributions}

K. Mochizuki designed the study, collected and analysed the data, and drafted the manuscript. K. Mori, HK, MI, KN, and $\mathrm{HI}$ analysed data. All authors participated in manuscript writing and approved the final version of the manuscript. All authors read and approved the final manuscript.

\section{Funding}

This research received no specific grant from any funding agency in the public, commercial, or not-for-profit sectors.

\section{Availability of data and materials}

The dataset analysed during the current study is available in Hayakawa M. et al. Nationwide registry of sepsis patients in Japan focused on disseminated intravascular coagulation 2011-2013, Scientific Data. 2018:5:180243. https:// doi.org/10.1038/sdata.2018.243.

\section{Ethics approval and consent to participate}

The study protocol complied with the principles of the Declaration of Helsinki and the Code of Ethics of the World Medical Association for experiments that involve humans. The study protocol was approved by the Ethics Committee of the Shinshu University School of Medicine, Matsumoto, Japan (\#4274). The dataset, which contained individual data of patients and institutions, had been anonymised prior to the analysis, and the Institutional Review Board decided that individual informed consent was not needed for the retrospective study.

\section{Consent for publication}

Not applicable.

\section{Competing interests}

K Mochizuki has received personal fees from Asahi Kasei Pharma Co. and Toray Medical Co., which were unrelated to the present study. All other authors declare that they have no conflicts of interest.

Received: 30 January 2020 Accepted: 4 May 2020

Published online: 13 May 2020

\section{References}

1. McColl T, Gatien M, Calder L, Yadav K, Tam R, Ong M, et al. Implementation of an emergency department sepsis bundle and system redesign: a process improvement initiative. CJEM. 2017;19:112-21.

2. Teles F, Rodrigues WG, Alves MGTC, Albuquerque CFT, Bastos SMO, Mota MFA, et al. Impact of a sepsis bundle in wards of a tertiary hospital. J Intensive Care. 2017;5:45.

3. Raj SK, Panda PK, Wig N, Agarwal P, Pandey RM. Compliance with 6 h-sepsis resuscitation bundle of Surviving Sepsis Campaign before and after resident physicians' training: a quality improvement interventional study among Indian patients. J Emerg Trauma Shock. 2019;12:3-9.

4. Rhodes A, Evans LE, Alhazzani W, Levy MM, Antonelli M, Ferrer R, et al. Surviving Sepsis Campaign: international guidelines for management of sepsis and septic shock: 2016. Intensive Care Med. 2017:43:304-77.

5. Singer M, Deutschman CS, Seymour CW, Shankar-Hari M, Annane D, Bauer $M$, et al. The third international consensus definitions for sepsis and septic shock (Sepsis-3). JAMA. 2016;315:801-10.

6. Cecconi M, Evans L, Levy M, Rhodes A. Sepsis and septic shock. Lancet. 2018:392:75-87.

7. Angus DC, van der Poll T. Severe sepsis and septic shock. N Engl J Med. 2013;369:840-51

8. Perner A, Gordon AC, Angus DC, Lamontagne F, Machado F, Russell JA, et al. The intensive care medicine research agenda on septic shock. Intensive Care Med. 2017:43:1294-305.

9. Gando S, Kameue T, Nanzaki S, Nakanishi Y. Disseminated intravascular coagulation is a frequent complication of systemic inflammatory response syndrome. Thromb Haemost. 1996;75:224-8.

10. Okamoto K, Tamura T, Sawatsubashi Y. Sepsis and disseminated intravascular coagulation. J Intensive Care. 2016;4:23.
11. Zeerleder S, Hack CE, Wuillemin WA. Disseminated intravascular coagulation in sepsis. Chest. 2005;128:2864-75.

12. Kudo D, Kushimoto S, Miyagawa N, Sato T, Hasegawa M, Ito F, et al. The impact of organ dysfunctions on mortality in patients with severe sepsis: a multicenter prospective observational study. J Crit Care. 2018;45:178-83.

13. Okabayashi K, Wada H, Ohta S, Shiku H, Nobori T, Maruyama K. Hemostatic markers and the sepsis-related organ failure assessment score in patients with disseminated intravascular coagulation in an intensive care unit. Am J Hematol. 2004;76:225-9.

14. Levi M, van der Poll T. Endothelial injury in sepsis. Intensive Care Med. 2013;39:1839-42.

15. Polderman $\mathrm{KH}$, Girbes AR. Drug intervention trials in sepsis: divergent results. Lancet. 2004;363:1721-3.

16. Umemura Y, Yamakawa K, Ogura H, Yuhara H, Fujimi S. Efficacy and safety of anticoagulant therapy in three specific populations with sepsis: a meta-analysis of randomized controlled trials. J Thromb Haemost. 2016;14:518-30.

17. Yatabe T, Inoue S, Sakamoto S, Sumi Y, Nishida O, Hayashida K, et al. The anticoagulant treatment for sepsis induced disseminated intravascular coagulation; network meta-analysis. Thromb Res. 2018;171:136-42.

18. Ito T, Thachil J, Asakura H, Levy JH, Iba T. Thrombomodulin in disseminated intravascular coagulation and other critical conditions-a multi-faceted anticoagulant protein with therapeutic potential. Crit Care. 2019;23:280.

19. Aikawa N, Shimazaki S, Yamamoto Y, Saito H, Maruyama I, Ohno R, et al. Thrombomodulin alfa in the treatment of infectious patients complicated by disseminated intravascular coagulation: subanalysis from the phase 3 trial. Shock. 2011;35:349-54.

20. Vincent JL, Ramesh MK, Ernest D, LaRosa SP, Pachl J, Aikawa N, et al. A randomized, double-blind, placebo-controlled, phase $2 \mathrm{~b}$ study to evaluate the safety and efficacy of recombinant human soluble thrombomodulin, ART-123, in patients with sepsis and suspected disseminated intravascular coagulation. Crit Care Med. 2013;41:2069-79.

21. Hagiwara A, Tanaka N, Uemura T, Matsuda W, Kimura A. Can recombinant human thrombomodulin increase survival among patients with severe septic-induced disseminated intravascular coagulation: a single-centre, open-label, randomised controlled trial. BMJ Open. 2016;6:e012850.

22. Mori S, Ai T, Sera T, Ochiai K, Otomo Y. Human soluble recombinant thrombomodulin, ART-123, resolved early phase coagulopathies, but did not significantly alter the 28 day outcome in the treatment of DIC associated with infectious systemic inflammatory response syndromes. J Clin Med. 2019;8:E1553.

23. Yamakawa K, Murao S, Aihara M. Recombinant human soluble thrombomodulin in sepsis-induced coagulopathy: an updated systematic review and meta-analysis. Thromb Haemost. 2019;119:56-65.

24. Yamakawa K, Levy JH, Iba T. Recombinant human soluble thrombomodulin in patients with sepsis-associated coagulopathy (SCARLET): an updated meta-analysis. Crit Care. 2019;23:302.

25. Vincent JL, Francois B, Zabolotskikh I, Daga MK, Lascarrou JB, Kirov MY, et al. Effect of a recombinant human soluble thrombomodulin on mortality in patients with sepsis-associated coagulopathy: the SCARLET randomized clinical trial. JAMA. 2019;321:1993-2002.

26. Danner RL, Elin RJ, Hosseini JM, Wesley RA, Reilly JM, Parillo JE. Endotoxemia in human septic shock. Chest. 1991;99:169-75.

27. Shoji H, Tani T, Hanasawa K, Kodama M. Extracorporeal endotoxin removal by polymyxin $B$ immobilized fiber cartridge: designing and antiendotoxin efficacy in the clinical application. Ther Apher. 1998:2:3-12

28. Ronco C, Klein DJ. Polymyxin B hemoperfusion: a mechanistic perspective. Crit Care. 2014;18:309.

29. Ono S, Tsujimoto H, Hiraki S, Aosasa S. Mechanisms of sepsis-induced immunosuppression and immunological modification therapies for sepsis. Ann Gastroenterol Surg. 2018;2:351-8.

30. Srisawat N, Tungsanga S, Lumlertgul N, Komaenthammasophon C, Peerapornratana S, Thamrongsat N, et al. The effect of polymyxin B hemoperfusion on modulation of human leukocyte antigen DR in severe sepsis patients. Crit Care. 2018;22:279. 
31. Cruz DN, Antonelli M, Fumagalli R, Foltran F, Brienza N, Donati A, et al. Early use of polymyxin B hemoperfusion in abdominal septic shock: the EUPHAS randomized controlled trial. JAMA. 2009;301:2445-52.

32. Payen DM, Guilhot J, Launey Y, Lukaszewicz AC, Kaaki M, Veber B, et al. Early use of polymyxin B hemoperfusion in patients with septic shock due to peritonitis: a multicenter randomized control trial. Intensive Care Med. 2015:41:975-84.

33. Dellinger RP, Bagshaw SM, Antonelli M, Foster DM, Klein DJ, Marshall JC, et al. Effect of targeted polymyxin B hemoperfusion on 28-day mortality in patients with septic shock and elevated endotoxin level: the EUPHRATES Randomized Clinical Trial. JAMA. 2018;320:1455-63.

34. Fujii T, Ganeko R, Kataoka Y, Furukawa TA, Featherstone R, Doi K, et al. Polymyxin B-immobilized hemoperfusion and mortality in critically ill adult patients with sepsis/septic shock: a systematic review with meta-analysis and trial sequential analysis. Intensive Care Med. 2018;44:167-78.

35. Hayakawa M, Yamakawa K, Saito S, Uchino S, Kudo D, lizuka Y, et al. Recombinant human soluble thrombomodulin and mortality in sepsisinduced disseminated intravascular coagulation. A multicentre retrospective study. Thromb Haemost. 2016;115:1157-66.

36. Nakamura Y, Kitamura T, Kiyomi F, Hayakawa M, Hoshino K, Kawano $Y$, et al. Potential survival benefit of polymyxin B hemoperfusion in patients with septic shock: a propensity-matched cohort study. Crit Care. 2017;21:134.

37. Yoshimura J, Yamakawa K, Ogura H, Umemura Y, Takahashi H, Morikawa $M$, et al. Benefit profile of recombinant human soluble thrombomodulin in sepsis-induced disseminated intravascular coagulation: a multicenter propensity score analysis. Crit Care. 2015;19:78.

38. Klein DJ, Foster D, Walker PM, Bagshaw SM, Mekonnen H, Antonelli M. Polymyxin B hemoperfusion in endotoxemic septic shock patients without extreme endotoxemia: a post hoc analysis of the EUPHRATES trial. Intensive Care Med. 2018;44:2205-12.

39. Yamato M, Minematsu Y, Fujii J, Mori K, Minato T, Miyagawa S, et al. Effective combination therapy of polymyxin-B direct hemoperfusion and recombinant thrombomodulin for septic shock accompanied by disseminated intravascular coagulation: a historical controlled trial. Ther Apher Dial. 2013;17:472-6.

40. Hayakawa M, Yamakawa K, Saito S, Uchino S, Kudo D, lizuka Y, et al. Nationwide registry of sepsis patients in Japan focused on disseminated intravascular coagulation 2011-2013. Sci Data. 2018;5:180243.

41. Bone RC, Balk RA, Cerra FB, Dellinger RP, Fein AM, Knaus WA, et al. Definitions for sepsis and organ failure and guidelines for the use of innovative therapies in sepsis. The ACCP/SCCM Consensus Conference Committee. American College of Chest Physicians/Society of Critical Care Medicine. Chest. 1992;101:1644-55

42. Gando S, Iba T, Eguchi Y, Ohtomo Y, Okamoto K, Koseki K, et al. A multicenter, prospective validation of disseminated intravascular coagulation diagnostic criteria for critically ill patients: comparing current criteria. Crit Care Med. 2006;34:625-31.

43. Gando S, Saitoh D, Ogura H, Mayumi T, Koseki K, Ikeda T, et al. Natural history of disseminated intravascular coagulation diagnosed based on the newly established diagnostic criteria for critically ill patients: results of a multicenter, prospective survey. Crit Care Med. 2008;36:145-50.

44. Austin PC. The performance of different propensity score methods for estimating marginal hazard ratios. Stat Med. 2013;32:2837-49.

45. Lee BK, Lessler J, Stuart EA. Improving propensity score weighting using machine learning. Stat Med. 2010;29:337-46.

46. Iba T, Levy JH. Inflammation and thrombosis: roles of neutrophils, platelets and endothelial cells and their interactions in thrombus formation during sepsis. JThromb Haemost. 2018;16:231-41.

47. Asakura H, Takahashi H, Tsuji H, Matsushita T, Ninomiya H, Honda G, et al. Post-marketing surveillance of thrombomodulin alfa, a novel treatment of disseminated intravascular coagulation — safety and efficacy in 1032 patients with hematologic malignancy. Thromb Res. 2014;133:364-70.

48. Taylor FB Jr, Toh CH, Hoots WK, Wada H, Levi M. Scientific Subcommittee on Disseminated Intravascular Coagulation (DIC) of the International Society on Thrombosis and Haemostasis (ISTH). Towards definition, clinical and laboratory criteria, and a scoring system for disseminated intravascular coagulation. Thromb Haemost. 2001;86:1327-30.

49. Singh RK, Baronia AK, Sahoo JN, Sharma S, Naval R, Pandey CM, et al. Prospective comparison of new Japanese Association for Acute Medicine (JAAM) DIC and international society of thrombosis and hemostasis (ISTH) DIC score in critically ill septic patients. Thromb Res. 2012;129:e119-25.

50. Gando S, Saitoh D, Ogura H, Fujishima S, Mayumi T, Araki T, et al. A multicenter, prospective validation study of the Japanese Association for Acute Medicine disseminated intravascular coagulation scoring system in patients with severe sepsis. Crit Care. 2013;17:R111.

\section{Publisher's Note}

Springer Nature remains neutral with regard to jurisdictional claims in published maps and institutional affiliations.

\section{Submit your manuscript to a SpringerOpen ${ }^{\odot}$ journal and benefit from:}

- Convenient online submission

- Rigorous peer review

- Open access: articles freely available online

- High visibility within the field

- Retaining the copyright to your article

Submit your next manuscript at springeropen.com 